\title{
HMGA1 is a novel transcriptional regulator of the FoxO1 gene
}

\author{
Biagio Arcidiacono $^{1} \cdot$ Eusebio Chiefari $^{1}{ }^{1} \cdot$ Sebastiano Messineo $^{1} \cdot$ \\ Francesco L. Bilotta $^{1} \cdot$ Ida Pastore $^{1} \cdot$ Domenica M. Corigliano $^{1} \cdot$ Daniela P. Foti $^{1}{ }^{1}$. \\ Antonio Brunetti ${ }^{1}{ }^{1}$
}

Received: 12 July 2017 / Accepted: 27 September 2017 / Published online: 19 October 2017

(C) The Author(s) 2017. This article is an open access publication

\begin{abstract}
Purpose The forkhead transcription factor (FoxO1) is a master transcriptional regulator of fundamental cellular processes ranging from cell proliferation and differentiation to inflammation and metabolism. However, despite its relevance, the mechanism(s) underlying FoxOl gene regulation are largely unknown. We have previously shown that the chromatin factor high-mobility group A1 (HMGA1) plays a key role in the transcriptional regulation of glucoseresponsive genes, including some that are involved in FoxO1-mediated glucose metabolism. Here we investigated the impact of HMGA1 on FoxOl gene expression.

Methods FoxO1 protein and gene expression studies were performed by Western blot analysis combined with qRTPCR of material from human cultured cells and EBVtransformed lymphoblasts, and from primary cultured hepatocytes from wild-type and $\mathrm{Hmgal}^{-/-}$mice. Reporter gene assays and chromatin immunoprecipitation for binding of HMGA1 to the endogenous FoxoOl locus were performed in cells overexpressing HMGA1 and in cells pretreated with siRNA targeting HMGA1.

Results HMGA1 increased FoxO1 mRNA and protein expression in vitro, in cultured HepG2 and HEK-293 cells by binding $\mathrm{FoxOl}$ gene promoter, thereby activating $\mathrm{FoxOl}$ gene transcription. Forced expression of HMGA1 in primary cultured hepatocytes from $\mathrm{Hmgal}^{-/-}$mice and in
\end{abstract}

Biagio Arcidiacono and Eusebio Chiefari contributed equally to this work.

Antonio Brunetti

brunetti@unicz.it

1 Department of Health Sciences, University "Magna Græcia" of Catanzaro, Viale Europa (Località Germaneto), 88100 Catanzaro, Italy
EBV-transformed lymphoblasts from subjects with reduced expression of endogenous HMGA1 increased FoxO1 mRNA and protein levels.

Conclusion These findings may contribute to the understanding of FoxOl gene regulation and its role in metabolism.

Keywords HMGA1 $\cdot$ FoxO1 $\cdot$ Insulin signaling $\cdot$ Gene transcription $\cdot$ DNA/chromatin interaction

\section{Introduction}

Forkhead box protein O1 (FoxO1), a member of the forkead family of transcription factors characterized by a highly conserved forkhead DNA-binding domain, regulates the transcription of genes involved in a wide spectrum of fundamental biological processes, like cell proliferation and differentiation, apoptosis and DNA repair, inflamation and stress response, insulin sensitivity and energy metabolism [1-4]. As a crucial effector of insulin action, FoxO1 is highly expressed in insulin-responsive tissues, including pancreas and liver, skeletal muscle, white and brown adipose tissue, skeleton and brain, at which levels FoxO1 exerts effects over glucose metabolism, insulin sensitivity and energy expenditure [5-9]. In particular, in the liver, FoxO1 stimulates gluconeogenic genes, such as phosphoenolpyruvate carboxykinase (PEPCK) and glucose-6phosphatase (G6Pase), as well as genes involved in glucose counterregulation, including the insulin-like growth factor binding protein 1 (IGFBPI) gene [10, 11], thus ensuring fasting euglycemia. After a meal, by triggering the phosphorylation of FoxO1 via AKT, insulin induces its 
detachment from DNA and its nuclear export, thereby inhibiting gluconeogenesis and suppressing hepatic glucose output [12-15]. Consequently, under insulin resistance conditions, FoxO1 phosphorylation is blunted, and the transcriptional activation of gluconeogenic genes is abnormally sustained, which confers increased susceptibilty to type 2 diabetes (T2D) mellitus [16].

While insulin regulation of FoxO1 protein is fairly well understood [17, 18] the mechanism(s) regulating FoxO1 gene expression have been poorly investigated and poorly documented, so that the only data available in this regard refer to the upregulation of FoxOl gene in response to E2F1 , a transcription factor involved in cell cycle progression and apoptosis [19], the homeodomain transcription factor ALX3 [20], FoxC1 [21], and FoxO1 itself [22]. Also, it has been reported that FoxO1 mRNA levels were increased in muscle of fasted or calorically restricted rats [23, 24], supporting the existence of a nutrient deprivation-induced mechanism activating FoxOl gene transcription. However, the transcription factors mediating this activation have not been identified.

The high-mobility group A1 (HMGA1) protein is a nuclear architectural factor that belongs to the superfamily of nonhistone chromatin-binding proteins. By binding to AT-rich regions of DNA, HMGA1 can transactivate gene promoters through mechanisms that facilitate the assembly and stability of stereospecific DNA-protein complexes (socalled enhanceosomes), thereby activating or repressing a variety of mammalian genes [25-27]. We previously showed that HMGA1 is an important novel mediator of glucose homeostasis in vivo, playing a crucial role in the transcriptional regulation of a variety of genes actively contributing to the maintenance of blood glucose levels, such as insulin [28] and the insulin receptor genes $[27,29,30]$, in addition to a series of insulin-target genes, including the gluconeogenic genes PEPCK and G6Pase [11], as well as genes encoding proteins that are involved in glucose counterregulation (e.g., IGFBP1 and the retinolbinding protein 4 (RBP4)) [11,31-33]. A role for HMGA1 as a nuclear target of insulin signaling has been reported by us before, showing that the insulin-mediated postprandial glucose disposal is mediated by detachment of HMGA1 from gluconeogenic gene promoters in a way that resembles the functional association between insulin and FoxO1 [11]. Accordingly, defects in HMGAl gene and protein expression have been linked to insulin resistance and T2D in humans and mice [30,34-38], whereas protection from these metabolic disorders has been reported in transgenic mice that overexpress HMGA1 [39].

Taking into account the above considerations, here we explored the possibility that HMGA1 may be involved in the transcriptional regulation of $\mathrm{FoxOl}$ gene.

\section{Materials and methods}

\section{Cell cultures, protein extracts and Western blot}

Human embryonic kidney 293 (HEK-293) cells and HepG2 human hepatoma cells were cultured in Dulbecco's modified Eagle medium and RPMI 1640 medium, respectively, supplemented with 10\% fetal bovine serum (FBS) (Gibco Laboratories, Grand Island, NY, USA), $2 \mathrm{mM}$ glutamine, penicillin $(100 \mathrm{U} / \mathrm{ml})$, and streptomycin $(100 \mu \mathrm{g} / \mathrm{ml})$ in a humidified $5 \% \mathrm{CO}_{2}$ atmosphere at $37^{\circ} \mathrm{C}$. Cryopreserved primary hepatocytes from $\mathrm{HMGA}^{-/-}$and wild-type mice [11] were cultured on matrigel-coated six-well plates in Williams E media (Sigma) supplemented with 10\% FBS and then utilized in subsequent experiments. Cultured lymphoblast cell lines transformed with Epstein-Barr virus (EBV) were from normal volunteers and previously identified diabetic subjects carrying HMGAl gene defects [30, 34, 40], and maintained in RPMI 1640 medium supplemented with $15 \%$ FBS. Nuclear protein extracts were prepared as previously [29] and final protein concentration in the extracts was determined by the modified Bradford method (Bio-Rad Laboratories, Hercules, CA, USA). The antibodies used for Western blot studies were: anti-HMGA1 [41], anti-FoxO1 (Santa Cruz Biotechnology, Santa Cruz, CA) and anti Sp1 [42].

\section{Plasmid construction and transfections}

Human FoxOl gene promoter region $(-1989$ to -155 bp relative to the start site of transcription) was amplified from the BAC clone RP11-181D10 (Invitrogen Life Technology Corporation, Carlsbad, Calif. USA), using modified specific primers. After sequence analysis, the resulting product was cloned upstream to the luciferase (Luc) gene into pGL3 basic vector (Promega, Madison, WI, USA), to obtain the recombinant plasmid FoxOl-Luc. This construct was transiently transfected into HepG2 or HEK-293 cells, using the LipofectAMINE 2000 reagent (Invitrogen), in the presence or absence of an effector vector (pcDNA3HA-HMGA1a) for the HMGA1a isoform protein, as obtained by subcloning the BamH1/EcoR1 fragment from pcDNA1-HMGI into the pcDNA3HA plasmid (Invitrogen) [27]. Luc activity was assayed $48 \mathrm{~h}$ later in a luminometer (Turner Biosystems Inc., CA, USA), using the dual-luciferase reporter assay system (Promega, Madison, Wis., USA). For gene silencing experiments, cells at $40-50 \%$ confluency were transfected with 100-200 pmol anti-HMGAl siRNA plus nonspecific control siRNA with a similar GC content (Santa Cruz Biotechnology, Santa Cruz, CA) and maintained in culture for $72 \mathrm{~h}$, after which cells were removed from the culture plates and subjected to a second transfection with the same 
siRNAs for an additional $72 \mathrm{~h}$. Renilla control vector served as an internal control of transfection efficiency. EBVtransformed lymphoblasts were transfected with expression vector containing the HMGA1 cDNA as described previously [27]. For the selection of transfected cells, lymphoblasts were cotransfected with the pEGFP vector (Clontech) and collected $18 \mathrm{~h}$ later with a FACS Vantage cell sorter (Becton Dickinson). Sorted cells were maintained in culture and used for gene and protein expression analyses $72 \mathrm{~h}$ later [26].

\section{Chromatin immunoprecipitation (ChIP)}

ChIP was performed in HepG2 and EBV-transformed lymphoblasts, either untreated or pre-treated with $H M G A 1$ siRNA or with HMGAl cDNA, as described previously [11, 30, 43]. Formaldehyde-fixed DNA-protein complex was immunoprecipitated with anti-HMGA1 antibody $[30,44]$, and the following primers for the FoxOl gene promoter were used for PCR amplification of ChIP-ed DNA (30 cycles), using PCR ready-to-go beads (Amersham Pharmacia Biotech): human FoxOl (NT_007819) for 5'CCCAAGGCTTTGGTCCTATC-3', rev 5'- GCCGGATTCACTGTATTCTTG $-3^{\prime}$. PCR products were electrophoretically resolved on $1.5 \%$ agarose gel and visualized by ethidium bromide staining.

\section{Real-time PCR}

For qRT-PCR, total cellular RNA was extracted from cells using the RNAqueous-4PCR kit and subjected to DNase treatment (Ambion). RNA levels were normalized against 18 $S$ ribosomal RNA in each sample, and cDNAs were synthesized from $2 \mu \mathrm{g}$ of total RNA using the RETROscript first strand synthesis kit (Ambion). Primers for human FoxOl (NM_002015.3) (5'- AAGGATAAGGGTGACAGCAACAG-3'; 5'- TTGCTGTGTAGGGACAGATTATGAC-3'), and mouse FoxOl (NM_019739) (5'- CAAAGTACACATACGGCCAATCC-3'; 5' - CGTAACTTGATTTGCTGTCC TGAA-3'), were designed according to sequences from the GenBank database. qRT-PCR was performed as previously described [32], using RPS9 cDNA as an internal standard. All PCR reactions were done in triplicates.

\section{Statistics}

Statistical significance was evaluated by Student's $t$ test. $P$ $<0.05$ was considered significant. All bar graph data shown are mean \pm S.E.M.

\section{Results}

\section{HMGA1 induces FoxO1 gene transcription}

To explore the possibility that HMGA1 could be involved in the regulation of FoxO1 expression, we first carried out in vitro studies in HepG2 and HEK-293 cells, two human cell lines which express high and low levels of endogenous HMGA1, respectively. Following transient transfections with either HMGA1 expression vector or siRNA targeting HMGA1, both FoxOl mRNA and protein levels were increased in HepG2 cells overexpressing HMGA1, and were reduced in cells pretreated with siRNA targeting HMGAl (Fig. 1a). Overexpression of increasing amounts of HMGA1 in HEK-293 cells increased FoxO1 mRNA and protein levels in a dose-dependent manner (Fig. 1b). To test the hypothesis that HMGA1 could play a role in the transcriptional regulation of the FoxOl gene, we performed reporter gene analysis in HepG2 and HEK-293 cells, after transfecting both cell lines with the FoxO1-Luc reporter plasmid bearing the human FoxOl promoter sequence upstream to the luciferase reporter gene, in the presence of increasing amounts of either siRNA targeting HMGAl or HMGA1 expression vector, respectively. As shown in Fig. 2a, FoxO1-Luc activity was reduced in HepG2 cells pretreated with siRNA targeting $H M G A 1$, whereas overexpression of HMGA1 in HEK-293 cells increased FoxO1$L u c$ activity in a dose-dependent manner and this effect was reduced in cells treated with distamycin A, a small molecule inhibitor of HMGA1 protein binding to DNA [11] (Fig. 2b), thus indicating that HMGA1 is required for the regulation of this promoter in vitro.

\section{HMGA1 binds FoxO1 gene promoter}

Based on the above functional observations and the presence of several putative binding sites for HMGA1 in the promoter region of $\mathrm{FoxO}$, as revealed by sequence analysis with MatInspector software (version 8.1, Genomatix, http:// www.genomatix.de/), we performed ChIP coupled with qRT-PCR of ChIP-ed samples, showing that binding of HMGA1 to the endogenous FoxOl chromosomal locus was manifest in living HepG2 cells naturally expressing HMGA1, and was decreased in cells that were exposed to distamycin A (Fig. 3). The reduction in HMGA1 occupancy at the endogenous FoxOl locus was also reduced by treating cells with siRNA against HMGA1 (Fig. 3), further substantiating the functional role of HMGA1 at the level of FoxOl gene promoter. Thus, taken together, these data demonstrate that HMGA1 is of major importance for the transcriptional regulation of the FoxOl gene, suggesting that functional impairment of HMGA1 may contribute to 
Fig. 1 FoxOl gene expression is induced by HMGA1. a pcDNA3HMGAl effector vector was transfected into HepG2 cells, either in the absence or presence of siRNA against HMGA1, or a non-targeting control siRNA, and endogenous FoxO1 mRNA expression was measured thereafter. b Increasing amounts $(0,0.5,1 \mu \mathrm{g})$ of pcDNA3HMGA1 effector vector were transfected into HEK-293 cells, and $\mathrm{FoxOl}$ mRNA was measured as in $\mathbf{a}$. Results are means \pm S.E.M. of three independent experiments, each in triplicate. $* P<0.05$ vs. control (first column in each condition). Representative Western blots (WB) of FoxO1 and HMGA1 out of three independent experiments for each condition are shown in the autoradiograms. Bar graphs above the gel panels are derived from densitometric scanning of WBs, using the ImageJ software program. $* P<0.05$ vs. controls (first columns in each condition). Sp1, control of nuclear protein loading
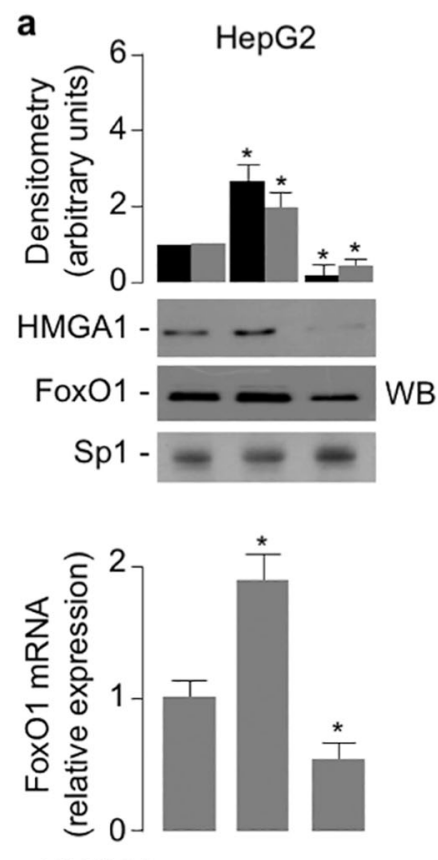

Effector: HMGA1 - + -

SiRNA: Control ++HMGA1 - -+ b
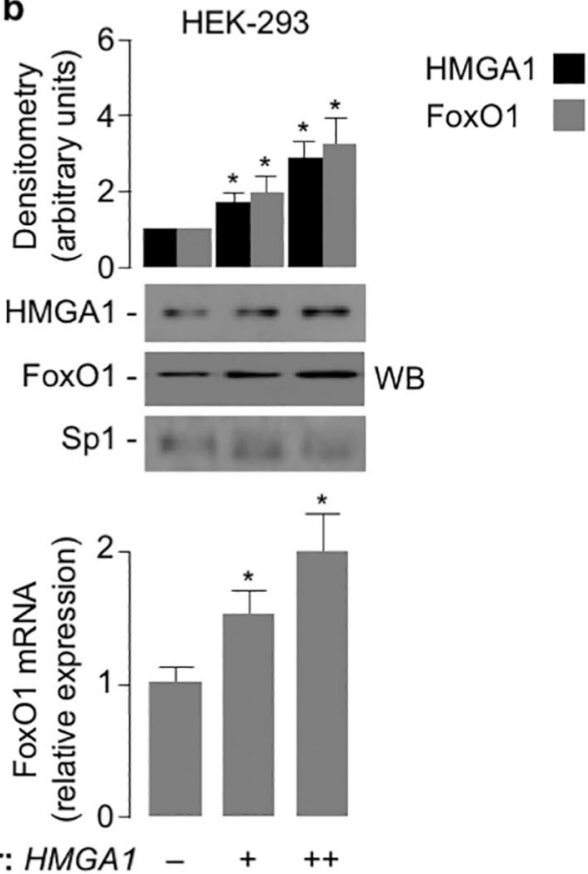

Effector: HMGA1 - + ++ reduced expression of FoxO1, thereby to the inhibition of FoxO1-mediated gene transcription and function (e.g., hepatic gluconeogenesis).

\section{FoxO1 gene expression in primary cultured hepatocytes from $\mathrm{Hmgal}^{-/-}$mice}

We previously showed that HMGA1 plays an essential role in the transcriptional activation of the FoxO1-dependent gluconeogenic genes PEPCK and G6Pase, as well as on the transcription of the insulin-target gene IGFBPI, thereby contributing to the maintenance of fasting euglycemia $[11,30]$. Also, consistently with our findings above and the role of FoxO1 in hepatic gluconeogenesis, plasma glucose concentration in $\mathrm{Hmgal}^{-/}$mice was lower than in wildtype animals [32], suggesting that HMGA1 may be required for $\mathrm{FoxOl}$ gene expression and function also in vivo, in whole animal. This hypothesis has now been further supported in the present work, by additional studies of primary cultured hepatocytes from $\mathrm{Hmgal}^{-/-}$and wild-type mice, showing that the mRNA expression of FoxO1 was lower in $\mathrm{Hmgal}^{-/-}$hepatocytes compared to wild-type hepatocytes (Fig. 4). Restoration of HMGA1 expression following transfection of primary cultured hepatocytes with a HMGA1 expression vector, enhanced mRNA levels for
FoxO1, thus further proving that HMGA1 is essential for FoxOl gene expression.

\section{FoxO1 is reduced in subjects carrying defects in HMGA1}

In the light of the above experimental results, we thought to investigate the expression of FoxO1 in individuals with variable amounts of HMGA1. To this end, FoxO1 expression levels were measured in cultured EBV-transformed lymphoblasts from previously selected individuals with and without insulin resistance and T2D, with and without HMGAl gene defects adversely affecting HMGA1 protein production [30,34-36]. As shown in Fig. 5, FoxO1 mRNA abundance was reduced in cells expressing low levels of HMGA1, and this reduction paralleled the decrease in FoxO1 protein expression, as detected by immunoblots from nuclear extracts of cultured lymphoblasts. Restoration of HMGA1 protein expression also in these subjects' cells enhanced $\mathrm{FoxOl}$ expression (Fig. 5), thus indicating that, as observed in murine primary hepatocytes, HMGA1 can also regulate $\mathrm{FoxOl}$ expression in human-derived cells. The role of HMGA1 on FoxOl promoter, in humans, was corroborated by ChIP experiments coupled with qRT-PCR of ChIPed DNA, showing that binding of HMGA1 to the endo- 


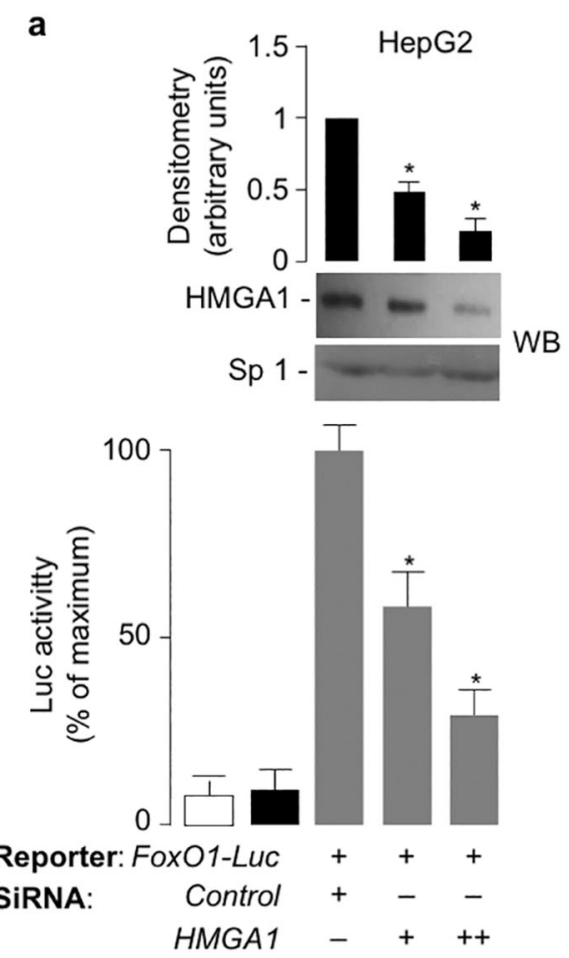

Fig. 2 HMGA1 regulates FoxO1 gene transcription. a FoxO1-Luc reporter vector was transfected into HepG2 cells, either in the absence or presence of siRNA against HMGA1, or a nontargeting control siRNA, and Luc activity was measured 72 to $96 \mathrm{~h}$ later. b FoxOl-Luc reporter vector was transfected into HEK-293 cells, with increasing amounts $(0,0.5,1 \mu \mathrm{g})$ of pcDNA3-HMGAl effector vector, in the absence or presence of $10 \mu \mathrm{M}$ distamycin A, and Luc activity was measured as in a. In both experimental conditions, Luc activity from the reporter plasmid was normalized by the renilla Luc activity produced from a pRL Renilla-Luc control vector cotransfected as an internal control. White bar, mock (no DNA); black bar, (pGL3 basic b
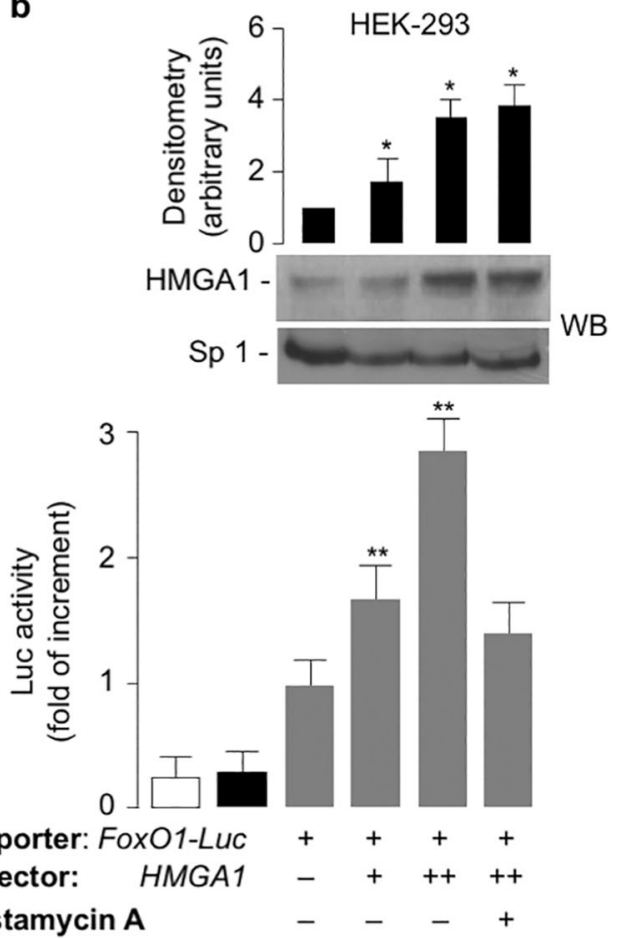

vector, without the FoxO1 promoter). Results are means \pm S.E.M. of three independent experiments, each in triplicate. $* P<0.05$ compared with nontargeting (control) siRNA; $* * P<0.05$ vs. the FoxO1-Luc, in the absence of a HMGA1 effector vector, which is assigned an arbitrary value of 1 . Representative WBs of HMGA1 out of three independent experiments for each condition are shown in the autoradiograms. Bar graphs above the gel panels are derived from densitometric scanning of WBs, using the ImageJ software program. ${ }^{*} P<0.05$ vs. control (first column in each condition). Sp1, control of nuclear protein loading

genous FoxOl chromosomal locus was low in living EBVtransformed cells from subjects with reduced expression of HMGA1, and was considerably increased in EBV cells after transfection of the expression vector for HMGA1 (Fig. 6).

\section{Discussion}

We previously provided data indicating that HMGA1 may play a fundamental role in insulin signaling and the transcriptional regulation of glucose metabolism [11,30-32]. This notion is based mainly on the observation that HMGA1 is a key regulator of a variety of insulin-target genes, including the insulin receptor gene, as well as the gluconeogenic genes PEPCK and G6Pase, in addition to other genes encoding for proteins important in glucose counterregulation and homeostasis such as, IGFBP1 and RBP4 [11, 31, 32]. 


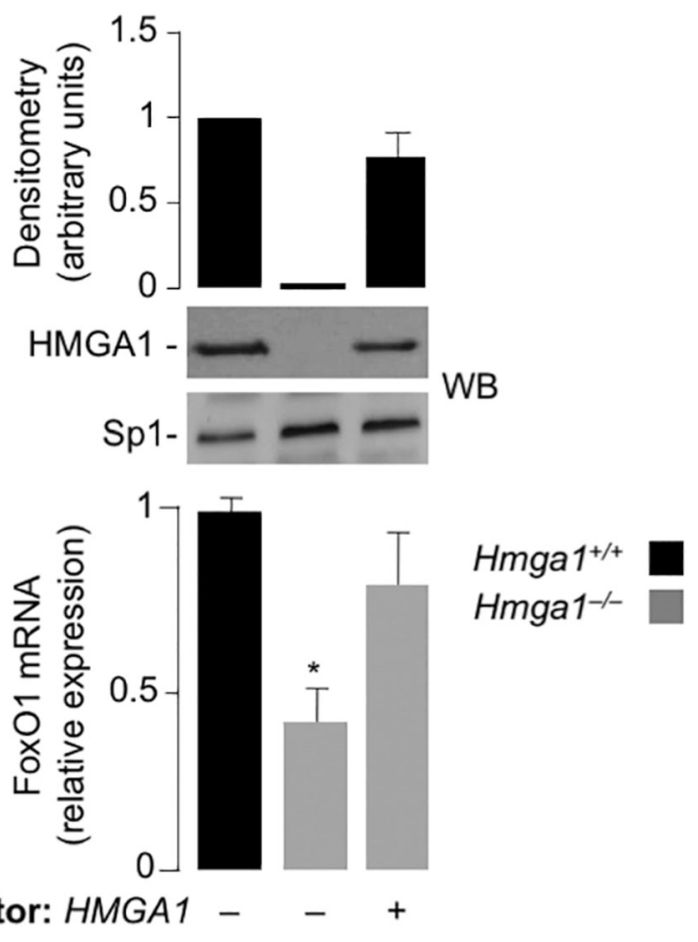

Fig. 4 FoxOl gene expression in primary culture cells. FoxO1 mRNA abundance was measured in primary cultured hepatocytes from both $\mathrm{Hmgal}^{+/+}$(black bar) and $\mathrm{Hmgal}^{-/-}$(gray bar) mice, before and after transfection of the cells with the pcDNA3-HMGAl expression vector. Results are means \pm S.E.M. of five independent experiments. $* P<0.05$ vs. Hmgal ${ }^{+/+}$hepatocytes. Representative WBs are shown in each condition. Bar graph above the gel panel is derived from densitometric scanning of anti-HMGA1 WB, using the ImageJ software program. Sp1, control of nuclear protein loading

On the other hand, the specific action of the transcription factor FoxO1 in regulating glucose homeostasis has been reported in several studies, which have also contributed significantly to our understanding of the mechanism of action and the physiological significance of FoxO1 in this context. For example, cytoplasm retention of FoxO1 via insulin-induced phosphorylation is suggested to be a mechanism of insulin-mediated gene repression, thus a critical regulator of peripheral insulin action [45-47]. The possibility that HMGA1, by affecting Foxol gene and protein expression can be a component of this regulation, would constitute an interesting finding of the present work, which, however, deserves further research to be better assessed. Compared to previous investigations, for the first time in the present study, we report the identification of HMGA1 as a novel nuclear modulator of the Foxol gene, which might play a major role in FoxO1-mediated transactivation in mammals.

In our study, the involvement of HMGA1 in the expression of FoxO1 is underlined by the following facts: first, HMGA1 enhances FoxOl gene expression and promoter activity in cultured cells; second, binding of HMGA1

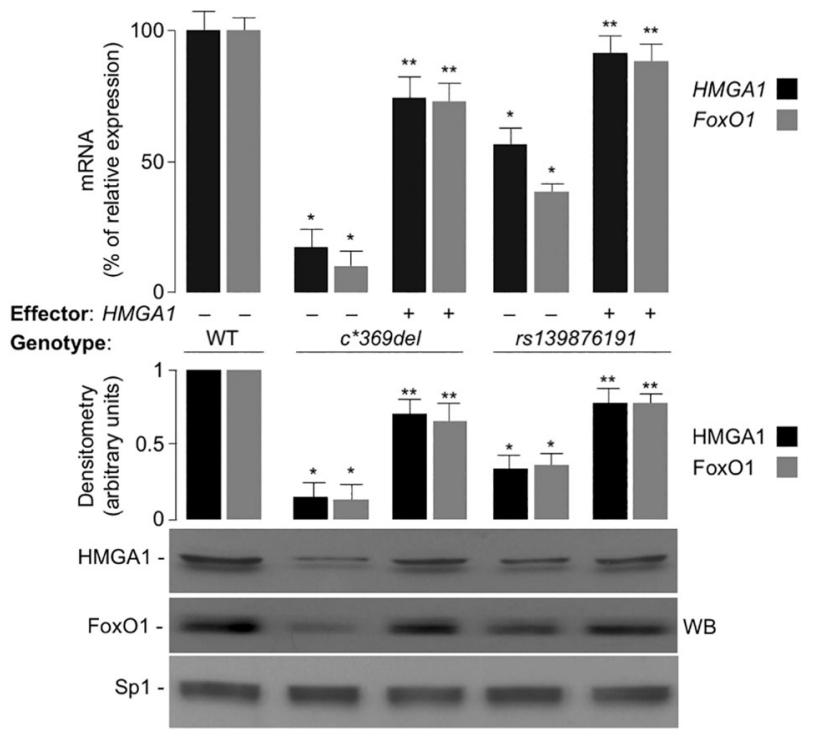

Fig. 5 FoxOl expression in EBV-transformed lymphoblasts from subjects expressing variable amounts of HMGA1. mRNA and protein expression (WB) for both HMGA1 and FoxO1 were concomitantly measured in cultured EBV-transformed lymphoblasts from normal subjects (WT, wild-type, $n=6$ ) and subjects carrying HMGAl gene variants $(c . * 369 \mathrm{del}$ and $r s 139876191 ; n=3$ and 4 , respectively) that decrease HMGA1 protein expression [30, 34], either in the absence (-) or presence $(+)$ of the effector vector for HMGA1. qRT-PCR of HMGA1 and FoxO1 mRNA levels are shown as percent of maximal value (WT, $100 \%$ ). Results are means \pm S.E.M. for three separate assays. $* P<0.05$ vs. controls (WT). ${ }^{*} P<<0.05$ vs. untransfected cells in each variant group. Representative WBs for HMGA1 and FoxO1 are shown. Bar graphs above the gel panels are derived from densitometric scanning of WBs, using the ImageJ software program. $* P<0.05$ vs. controls (WT); $* * P<0.05$ vs. untransfected cells in each variant group. Sp1, control of nuclear protein loading

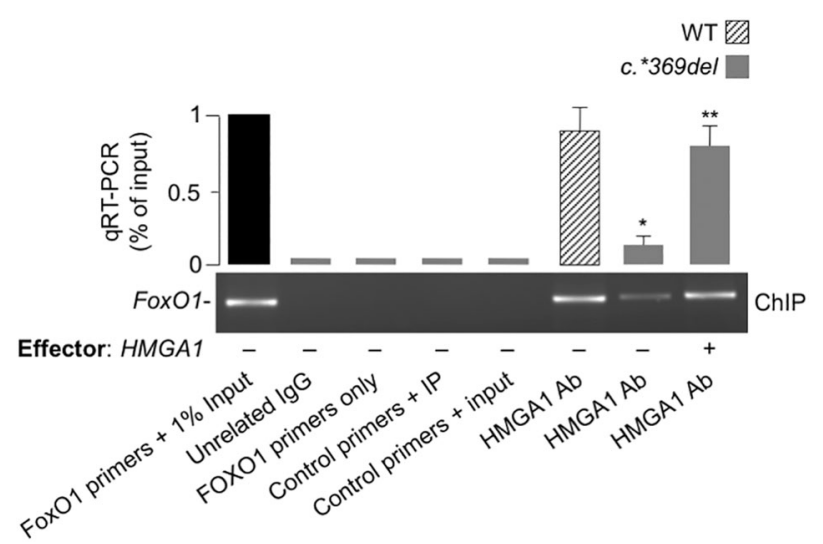

Fig. 6 Binding of HMGA1 to FoxOl promoter in subjects' cells. The occupancy of the FoxO1 gene promoter by HMGA1 was measured by ChIP in EBV-transformed lymphoblasts from normal WT subjects (slashed bar, $n=3$ ) and subjects carrying the HMGAl c.*369del variant (gray bars, $n=3$ ), using an anti-HMGA1 specific antibody $(\mathrm{Ab})$, either before or after transfecting cells with the c.*369del variant with an HMGA1 effector vector. qRT-PCRs of ChIP-ed samples are shown in each condition. ${ }^{*} P<0.05$ vs. WT subjects; $* * P<0.05$ vs. untransfected cells carrying the HMGAl c.*369del variant. A representative ChIP assay is presented 
to the endogenous FoxOl chromosomal locus is increased in living cells naturally expressing HMGA1, and is reduced in cells exposed either to distamycin A or siRNA against HMGA1; third, FoxO1 abundance is low in both primary cultured hepatocytes from $\mathrm{Hmgal}^{-/}$mice and EBVtransformed lymphoblasts from subjects with reduced expression of HMGA1, in which restoration of HMGA1 protein production enhances $\mathrm{FoxOl}$ gene and protein expression. Altogether, these observations strengthen the role of HMGA1 as a novel transcriptional activator of FoxO1 gene, lending support to the view that HMGA1, which is upregulated via the cAMP-signaling pathway during fast $[32,33]$, may play a pivotal role in maintaining fasting euglycemia, in vivo. Also, as concomitant insulin resistance and insulin hypersensitivity in peripheral tissues may paradoxically coexist in certain adverse metabolic conditions in which insulin action is precluded [30, 32], it is tempting to hypothesize that the reduction of HMGA1, by adversely affecting FoxO1 expression, might reflect an adaptive mechanism to increase insulin action.

From a clinical point of view, the observation that diabetic subjects with $H M G A 1$ gene defects might display also a decrease in FoxO1 protein expression supports the notion that patients with T2D who have these defects may have a different clinical course than other patients with T2D. In this respect, we recently reported that diabetic patients carrying the rs139876191 HMGAl variant (which causes reduced HMGA1 protein expression) had a significantly lower risk of proliferative diabetic retinopathy, compared to non-carrier patients, and this protective effect was attributed, at least in part, to downregulation of the vascular endothelial growth factor A, a major activator of neovascularization [48]. In this context, the involvement of FoxO1 in the induction of genes involved in diabetic retinopathy and its activation during the early inflammatory phase of diabetic retinopathy has been reported $[49,50]$. Thus, the protective effect of the HMGAl rs139876191 variant on diabetic retinopathy may also be mediated through a process that involves inactivation of $\mathrm{FoxOl}$ gene and protein expression. Although the relevance of our data to the clinical setting requires further investigation, they provide compelling evidence for the identification of HMGA1 as a novel nuclear activator of $\mathrm{FoxOl}$ gene transcription, which may help explaining noted differences in diabetes phenotypes.

In our opinion, these findings offer new biological and mechanistic insights into the mechanism(s) underlying FoxOl gene regulation and might be useful in understanding the molecular basis of clinical phenotypes in those conditions where insulin action is compromised (e.g., T2D and other insulin-resistant states). Understanding these mechanisms could help in identifying novel therapeutic targets for preventing and treating these diseases.

\section{Compliance with ethical standards}

Conflict of interest The authors declare that they have no competing interests.

Ethical approval This article does not contain any studies with human participants or animals performed by any of the authors.

Informed consent For this type of study formal consent is not required.

Open Access This article is distributed under the terms of the Creative Commons Attribution 4.0 International License (http://crea tivecommons.org/licenses/by/4.0/), which permits unrestricted use, distribution, and reproduction in any medium, provided you give appropriate credit to the original author(s) and the source, provide a link to the Creative Commons license, and indicate if changes were made.

\section{References}

1. M. Monsalve, Y. Olmos, The complex biology of FOXO. Curr. Drug Targets 12, 1322-1350 (2011)

2. D. Accili, K.C. Arden, FoxOs at the crossroads of cellular metabolism, differentiation, and transformation. Cell 117, 421-426 (2004)

3. M.L. Hribal, J. Nakae, T. Kitamura, J.R. Shutter, D. Accili, Regulation of insulin-like growth factor-dependent myoblast differentiation by Foxo forkhead transcription factors. J. Cell Biol. 162, 535-541 (2003)

4. J. Nakae, T. Kitamura, Y. Kitamura, W.H. Biggs 3rd, K.C. Arden, D. Accili, The forkhead transcription factor Foxo1 regulates adipocyte differentiation. Dev. Cell 4, 119-129 (2003)

5. S. Kousteni, FoxO1, the transcriptional chief of staff of energy metabolism. Bone 50, 437-443 (2012)

6. P. Puigserver, J. Rhee, J. Donovan, C.J. Walkey, J.C. Yoon, F. Oriente, Y. Kitamura, J. Altomonte, H. Dong, D. Accili, B.M. Spiegelman, Insulin-regulated hepatic gluconeogenesis through FOXO1-PGC-1alpha interaction. Nature 423, 550-555 (2003)

7. M.S. Kim, Y.K. Pak, P.G. Jang, C. Namkoong, Y.S. Choi, J.C. Won, K.S. Kim, S.W. Kim, H.S. Kim, J.Y. Park, Y.B. Kim, K.U. Lee, Role of hypothalamic Foxo1 in the regulation of food intake and energy homeostasis. Nat. Neurosci. 9, 901-906 (2006)

8. Y.I. Kitamura, T. Kitamura, J.P. Kruse, J.C. Raum, R. Stein, W. $\mathrm{Gu}, \mathrm{D}$. Accili, FoxO1 protects against pancreatic beta cell failure through NeuroD and MafA induction. Cell Metab. 2, 153-163 (2005)

9. M. Matsumoto, A. Pocai, L. Rossetti, R.A. Depinho, D. Accili, Impaired regulation of hepatic glucose production in mice lacking the forkhead transcription factor foxo1 in liver. Cell. Metab. 6, 208-216 (2007)

10. P.D.K. Lee, C.A. Conover, D.R. Powell, Regulation and function of insulinlike growth factor-binding protein-1. Proc. Soc. Exp. Biol. Med. 204, 4-29 (1993)

11. E. Chiefari, M.T. Nevolo, B. Arcidiacono, E. Maurizio, A. Nocera, S. Iiritano, R. Sgarra, K. Possidente, C. Palmieri, F. Paonessa, G. Brunetti, G. Manfioletti, D. Foti, A. Brunetti, HMGA1 is a novel downstream nuclear target of the insulin receptor signaling pathway. Sci. Rep. 2, 251 (2012). https://doi. org/10.1038/srep00251

12. W.H. Biggs 3rd, J. Meisenhelder, T. Hunter, W.K. Cavenee, K.C. Arden, Protein kinase B/Akt-mediated phosphorylation promotes 
nuclear exclusion of the winged helix transcription factor FKHR1. Proc. Natl. Acad. Sci. USA 96, 7421-7426 (1999)

13. H. Matsuzaki, H. Daitoku, M. Hatta, K. Tanaka, A. Fukamizu, Insulin-induced phosphorylation of FKHR (FOXO1) targets to proteosomal degradation. Proc. Natl. Acad. Sci. USA 100, 11285-11290 (2003)

14. G. Rena, A.R. Prescott, S. Guo, P. Cohen, T.G. Unterman, Roles of the forkhead in rhabdomyosarcoma (FKHR) phosphorylation sites in regulating 14-3-3 binding, transactivation and nuclear targeting. Biochem. J. 354, 605-612 (2001)

15. X. Zhang, L. Gan, H. Pan, S. Guo, X. He, S.T. Olson, A. Mesecar, S. Adam, T.G. Unterman, Phosphorylation of serine 256 suppresses transactivation by FKHR (FOXO1) by multiple mechanisms. Direct and indirect effects on nuclear/cytoplasmic shuttling and DNA binding. J. Biol. Chem. 277, 45276-45284 (2002)

16. U.B. Pajvani, D. Accili, The new biology of diabetes. Diabetologia 58, 2459-2468 (2015)

17. X.C. Dong, K.D. Copps, S. Guo, Y. Li, R. Kollipara, R.A. DePinho, M.F. White, Inactivation of hepatic Foxol by insulin signaling is required for adaptive nutrient homeostasis and endocrine growth regulation. Cell Metab. 8, 65-76 (2008)

18. V.T. Samuel, C.S. Choi, T.G. Phillips, A.J. Romanelli, J.G. Geisler, S. Bhanot, R. McKay, B. Monia, J.R. Shutter, R.A. Lindberg, G.I. Shulman, M.M. Veniant, Targeting foxo1 in mice using antisense oligonucleotide improves hepatic and peripheral insulin action. Diabetes 55, 2042-2050 (2006)

19. K. Nowak, K. Killmer, C. Gessner, W. Lutz, E2F-1 regulates expression of FOXO1 and FOXO3a. Biochim. Biophys. Acta 1769, 244-252 (2007)

20. P. García-Sanz, M. Mirasierra, R. Moratalla, M. Vallejo, Embryonic defence mechanisms against glucose-dependent oxidative stress require enhanced expression of Alx3 to prevent malformations during diabetic pregnancy. Sci. Rep. 7, 389 (2017)

21. F.B. Berry, J.M. Skarie, F. Mirzayans, Y. Fortin, T.J. Hudson, V. Raymond, B.A. Link, M.A. Walter, FOXC1 is required for cell viability and resistance to oxidative stress in the eye through the transcriptional regulation of FOXO1A. Hum. Mol. Genet. 17, 490-505 (2008)

22. A. Essaghir, N. Dif, C.Y. Marbehant, P.J. Coffer, J.B. Demoulin, The transcription of FOXO genes is stimulated by FOXO3 and repressed by growth factors. J. Biol. Chem. 284, 10334-10342 (2009)

23. T. Furuyama, H. Yamashita, K. Kitayama, Y. Higami, I. Shimokawa, N. Mori, Effects of aging and caloric restriction on the gene expression of Foxo1, 3, and 4 (FKHR, FKHRL1, and AFX) in the rat skeletal muscles. Microsc. Res. Tech. 59, 331-334 (2002)

24. M. Imae, Z. Fu, A. Yoshida, T. Noguchi, H. Kato, Nutritional and hormonal factors control the gene expression of FoxOs, the mammalian homologues of DAF-16. J. Mol. Endocrinol. 30, 253-262 (2003)

25. R. Reeves, Molecular biology of HMGA proteins: hubs of nuclear function. Gene 277, 63-81 (2001)

26. R. Sgarra, S. Zammitti, A. Lo Sardo, E. Maurizio, L. Arnoldo, S. Pegoraro, V. Giancotti, G. Manfioletti, HMGA molecular network: from transcriptional regulation to chromatin remodeling. Biochim. Biophys. Acta 1799, 37-47 (2010)

27. D. Foti, R. Iuliano, E. Chiefari, A. Brunetti, A nucleoprotein complex containing Sp1, C/EBP $\beta$, and HMGI-Y controls human insulin receptor gene transcription. Mol. Cell Biol. 23, 2720-2732 (2003)

28. B. Arcidiacono, S. Iiritano, E. Chiefari, F.S. Brunetti, G. Gu, D.P. Foti, A. Brunetti, Cooperation between HMGA1, PDX-1 and MafA is essential for glucose-induced insulin transcription in pancreatic beta cells. Front. Endocrinol. 5, 237 (2015). https://doi. org/10.3389/fendo.2014.00237
29. A. Brunetti, G. Manfioletti, E. Chiefari, I.D. Goldfine, D. Foti, Transcriptional regulation of human insulin receptor gene by the high-mobility group protein HMGI(Y). FASEB J. 15, 492-500 (2001)

30. D. Foti, E. Chiefari, M. Fedele, R. Iuliano, L. Brunetti, F. Paonessa, G. Manfioletti, F. Barbetti, A. Brunetti, C.M. Croce, A. Fusco, A. Brunetti, Lack of the architectural factor HMGA1 causes insulin resistance and diabetes in humans and mice. Nat. Med. 11, 765-773 (2005)

31. S. Iiritano, E. Chiefari, V. Ventura, B. Arcidiacono, K. Possidente, A. Nocera, M.T. Nevolo, M. Fedele, A. Greco, M. Greco, G. Brunetti, A. Fusco, D. Foti, A. Brunetti, The HMGA1-IGF-I/ IGFBP system: a novel pathway for modulating glucose uptake. Mol. Endocrinol. 26, 1578-1589 (2012)

32. E. Chiefari, F. Paonessa, S. Iiritano, I. Le Pera, D. Palmieri, G Brunetti, A. Lupo, V. Colantuoni, D. Foti, E. Gulletta, G. De Sarro, A. Fusco, A. Brunetti, The cAMP-HMGA1-RBP4 system: a novel biochemical pathway for modulating glucose homeostasis. BMC Biol. 7, 24 (2009). https://doi.org/10.1186/1741-7007-7-24

33. A. Bianconcini, A. Lupo, S. Capone, L. Quadro, M. Monti, D. Zurlo, A. Fucci, L. Sabatino, A. Brunetti, E. Chiefari, M.E. Gottesman, W.S. Blaner, V. Colantuoni, Transcriptional activity of the murine retinol-binding protein gene is regulated by a multiprotein complex containing HMGA1, p54 nrb/NonO, protein-associated splicing factor (PSF) and steroidogenic factor 1 (SF1)/liver receptor homologue 1 (LRH-1). Int. J. Biochem. Cell Biol. 41, 2189-2203 (2009)

34. E. Chiefari, S. Tanyolaç, F. Paonessa, C.R. Pullinger, C. Capula, S. Iiritano, T. Mazza, M. Forlin, A. Fusco, V. Durlach, A. Durlach, M.J. Malloy, J.P. Kane, S.W. Heiner, M. Filocamo, D.P. Foti, I.D. Goldfine, A. Brunetti, Functional variants of the HMGA1 gene and type 2 diabetes mellitus. JAMA 305, 903-912 (2011)

35. C.R. Pullinger, I.D. Goldfine, S. Tanyolaç, I. Movsesyan, M. Faynboym, V. Durlach, E. Chiefari, D.P. Foti, P.H. Frost, M.J. Malloy, A. Brunetti, J.P. Kane, Evidence that an HMGA1 gene variant associates with type 2 diabetes, body mass index, and high-density lipoprotein cholesterol in a Hispanic-American population. Metab. Syndr. Relat. Disord. 12, 25-30 (2014)

36. E. Chiefari, S. Tanyolaç, S. Iiritano, A. Sciacqua, C. Capula, B. Arcidiacono, A. Nocera, K. Possidente, F. Baudi, V. Ventura, G. Brunetti, F.S. Brunetti, R. Vero, R. Maio, M. Greco, M. Pavia, U. Hodoglugil, V. Durlach, C.R. Pullinger, I.D. Goldfine, F. Perticone, D. Foti, A. Brunetti, A polymorphism of HMGA1 is associated with increased risk of metabolic syndrome and related components. Sci. Rep. 3, 1491 (2013). https://doi.org/10.1038/ srep01491. (2013)

37. A. Bianco, E. Chiefari, C.G.A. Nobile, D.P. Foti, M. Pavia, A. Brunetti, The association between HMGA1 rs146052672 variant and type 2 diabetes: a transethnic meta-analysis. PLoS One 10, e0136077 (2015). https://doi.org/10.1371/journal.pone.0136077

38. E. Chiefari, S. Iiritano, F. Paonessa, I. Le Pera, B. Arcidiacono, M. Filocamo, D. Foti, S.A. Liebhaber, A. Brunetti, Pseudogenemediated posttranscriptional silencing of HMGA1 can result in insulin resistance and type 2 diabetes. Nat. Commun. 1, 40 (2010). https://doi.org/10.1038/ncomms 1040

39. A. Arce-Cerezo, M. García, A. Rodríguez-Nuevo, M. CrosaBonell, N. Enguix, A. Peró, S. Muñoz, C. Roca, D. Ramos, S. Franckhauser, I. Elias, T. Ferre, A. Pujol, J. Ruberte, J.A. Villena, F. Bosch, E. Riu, HMGA1 overexpression in adipose tissue impairs adipogenesis and prevents diet-induced obesity and insulin resistance. Sci. Rep. 5, 14487 (2015)

40. A. Brunetti, L. Brunetti, D. Foti, D. Accili, I.D. Goldfine, Human diabetes associated with defects in nuclear regulatory proteins for the insulin receptor gene. J. Clin. Invest. 97, 258-262 (1996) 
41. E. Chiefari, B. Arcidiacono, K. Possidente, S. Iiritano, V. Ventura, R. Pandolfo, F.S. Brunetti, M. Greco, D. Foti, A. Brunetti, Transcriptional regulation of the HMGA1 gene by octamerbinding proteins Oct-1 and Oct-2. PLoS One 8, e83969 (2013). https://doi.org/10.1371/journal.pone.0083969

42. S. Messineo, A.E. Laria, B. Arcidiacono, E. Chiefari, R.M. Luque Huertas, D.P. Foti, A. Brunetti, Cooperation between HMGA1 and HIF-1 contributes to hypoxia-induced VEGF and Visfatin gene expression in 3T3-L1 adipocytes. Front. Endocrinol. 7, 73 (2016). https://doi.org/10.3389/fendo.2016.00073

43. V. Costa, D. Foti, F. Paonessa, E. Chiefari, L. Palaia, G. Brunetti, E. Gulletta, A. Fusco, A. Brunetti, The insulin receptor: a new anticancer target for peroxisome proliferator-activated receptor- $\gamma$ $(\operatorname{PPAR} \gamma)$ and thiazolidinedione-PPAR $\gamma$ agonists. Endocr. Relat. Cancer 15, 325-335 (2008)

44. K. Gerrish, M.A. Cissell, R. Stein, The role of hepatic nuclear factor 1a and PDX-1 in transcriptional regulation of the pdx-1 gene. J. Biol. Chem. 276, 47775-47784 (2001)

45. B. Desvergne, L. Michalik, W. Wahli, Transcriptional regulation of metabolism. Physiol. Rev. 86, 465-514 (2006)
46. M.P. Czech, Insulin's expanding control of forkheads. Proc. Natl. Acad. Sci. USA 100, 11198-11200 (2003)

47. O. Puig, R. Tjian, Transcriptional feedback control of insulin receptor by dFOXO/FOXO1. Genes Dev. 19, 2435-2446 (2005)

48. E. Chiefari, V. Ventura, C. Capula, G. Randazzo, V. Scorcia, M. Fedele, B. Arcidiacono, M.T. Nevolo, F.L. Bilotta, M. Vitiello, C. Palmieri, E. Gulletta, A. Fusco, D. Foti, R. Vero, A. Brunetti, A polymorphism of HMGA1 protects against proliferative diabetic retinopathy by impairing HMGA1-induced VEGFA expression. Sci Rep. 6, 39429 (2016). https://doi.org/10.1038/srep39429

49. B. Ponugoti, G. Dong, D.T. Graves, Role of forkhead transcription factors in diabetes-induced oxidative stress. Exp. Diabetes Res. 2012, 939751 (2012). https://doi.org/10.1155/2012/ 939751

50. Y. Behl, P. Krothapalli, T. Desta, S. Roy, D.T. Graves, FOXO1 plays an important role in enhanced microvascular cell apoptosis and microvascular cell loss in type 1 and type 2 diabetic rats. Diabetes 58, 917-925 (2009) 